\title{
ON THE EQUATIONS OF EXTENSIONAL MOTION OF CRYSTAL PLATES*
}

\author{
BY \\ R. D. MINDLIN AND H. L. COOPER** \\ Columbia University
}

1. Introduction. The Cauchy-Voigt [1] equations of extensional motion of anisotropic, elastic plates may be written as

$$
\begin{aligned}
& \left(L_{1}+\rho \Omega^{2}\right) u+L_{5} w=0, \\
& L_{5} u+\left(L_{3}+\rho \Omega^{2}\right) w=0,
\end{aligned}
$$

where $u$ and $w$ are the components of displacement in the plane of $x$ and $z$ (the plane of the plate), $\Omega=i \partial / \partial t$ and the $L_{p}, p=1,3,5$, are the quadratic operators,

$$
\begin{aligned}
& L_{1}=\gamma_{11} \frac{\partial^{2}}{\partial x^{2}}+2 \gamma_{15} \frac{\partial^{2}}{\partial x \partial z}+\gamma_{55} \frac{\partial^{2}}{\partial z^{2}}, \\
& L_{3}=\gamma_{55} \frac{\partial^{2}}{\partial x^{2}}+2 \gamma_{35} \frac{\partial^{2}}{\partial x \partial z}+\gamma_{33} \frac{\partial^{2}}{\partial z^{2}}, \\
& L_{5}=\gamma_{15} \frac{\partial^{2}}{\partial x^{2}}+\left(\gamma_{13}+\gamma_{55}\right) \frac{\partial^{2}}{\partial x \partial z}+\gamma_{35} \frac{\partial^{2}}{\partial z^{2}} .
\end{aligned}
$$

In (2), the $\gamma_{p q}$ are the elastic stiffnesses relating the stress-resultants $\left(\tau_{p}\right)$ and the strains $\left(\epsilon_{a}\right)$ according 'to

where

$$
\tau_{p}=\gamma_{p q} \epsilon_{q} ; \quad \gamma_{p q}=\gamma_{a p} ; \quad p, q=1,3,5,
$$

$$
\epsilon_{1}=\frac{\partial u}{\partial x}, \quad \epsilon_{3}=\frac{\partial w}{\partial z}, \quad \epsilon_{5}=\frac{\partial u}{\partial z}+\frac{\partial w}{\partial x} .
$$

In the case of an isotropic plate,

$$
\gamma_{11}=\gamma_{33}, \quad 2 \gamma_{55}=\gamma_{11}-\gamma_{13}, \quad \gamma_{15}=\gamma_{35}=0
$$

and Lamé's [2] representation

$$
u=\frac{\partial \varphi}{\partial x}-\frac{\partial \psi}{\partial z}, \quad w=\frac{\partial \varphi}{\partial z}+\frac{\partial \psi}{\partial x}
$$

reduces solutions of the displacement-equations of motion to solutions of

$$
\gamma_{11} \nabla^{2} \varphi+\rho \Omega^{2} \varphi=0, \quad \gamma_{55} \nabla^{2} \psi+\rho \Omega^{2} \psi=0 .
$$

In a study of the three-dimensional equations of anisotropic elasticity, G. F. Carrier [3] considered a special elastic symmetry which, in the present context, corresponds to an orthotropic plate $\left(\gamma_{15}=\gamma_{35}=0\right)$ with the additional condition

$$
\left(\gamma_{11}-\gamma_{55}\right)\left(\gamma_{33}-\gamma_{55}\right)=\left(\gamma_{13}+\gamma_{55}\right)^{2}
$$

${ }^{*}$ Received September 12, 1960.

**Now at Queens College, New York, N. Y. 
and showed that, if

$$
\begin{aligned}
& u=\left(\gamma_{11}-\gamma_{55}\right)^{1 / 2} \frac{\partial \varphi}{\partial x}-\left(\gamma_{33}-\gamma_{55}\right)^{1 / 2} \frac{\partial \psi}{\partial z}, \\
& w=\left(\gamma_{33}-\gamma_{55}\right)^{1 / 2} \frac{\partial \varphi}{\partial z}+\left(\gamma_{11}-\gamma_{55}\right)^{1 / 2} \frac{\partial \psi}{\partial x},
\end{aligned}
$$

solutions of

$$
\gamma_{11} \frac{\partial^{2} \varphi}{\partial x^{2}}+\gamma_{33} \frac{\partial^{2} \varphi}{\partial z^{2}}+\rho \Omega^{2} \varphi=0, \quad \gamma_{55} \nabla^{2} \psi+\rho \Omega^{2} \psi=0
$$

yield, through (7), solutions of the displacement equations of motion.

In this paper, we find the most general representation, of this type, for the CauchyVoigt equations; and we show, with the example of quartz, how to find orientations of crystal plates which have constants of elasticity that satisfy the conditions required for reduction of the equations of motion to the simpler forms.

2. Generalization of Carrier's representation in two dimensions. Let

$$
\begin{gathered}
u=\left(a_{11} \frac{\partial}{\partial x}+a_{13} \frac{\partial}{\partial z}\right) \chi \equiv M_{1} \chi, \\
w=\left(a_{31} \frac{\partial}{\partial x}+a_{33} \frac{\partial}{\partial z}\right) \chi \equiv M_{3} \chi,
\end{gathered}
$$

expecting that there will be two sets of constants $a_{i i}$ and, hence, two functions $\chi$. Then (1) become

$$
\left[\left(L_{1}+\rho \Omega^{2}\right) M_{1}+L_{5} M_{3}\right] \chi=0, \quad\left[\left(L_{3}+\rho \Omega^{2}\right) M_{3}+L_{5} M_{1}\right] \chi=0 .
$$

Because of the presence of the term $\rho \Omega^{2}$, the only linear operator that can be a factor of the operator in brackets in the first of (10) is $M_{1}$ and in the second of (10) is $M_{3}$. Hence, if the bracketed operators in (10) are each to be expressed as a product of a linear and a quadratic operator, $M_{1}$ must be a factor of $L_{5} M_{3}$ and $M_{3}$ must be a factor of $L_{5} M_{1}$. Accordingly, we set

$$
M_{1} M_{3}=L_{5} \text {. }
$$

Upon equating coefficients of like derivatives in (11), we find

$$
a_{11} a_{31}=\gamma_{15}, \quad a_{33} a_{13}=\gamma_{35}, \quad a_{11} a_{33}+a_{13} a_{31}=\gamma_{13}+\gamma_{55} .
$$

If (12) are satisfied, (10) become

$$
M_{1}\left(L_{1}+M_{3}^{2}+\rho \Omega^{2}\right) \chi=0, \quad M_{3}\left(L_{3}+M_{1}^{2}+\rho \Omega^{2}\right) \chi=0 .
$$

Now, we require the quadratic operators to be the same in both of (13). Hence, we set

$$
L_{1}+M_{3}^{2}=L_{3}+M_{1}^{2}=N, \text { say, }
$$

where

$$
N=b_{1} \frac{\partial^{2}}{\partial x^{2}}+2 b_{5} \frac{\partial^{2}}{\partial x \partial z}+b_{3} \frac{\partial^{2}}{\partial z^{2}}
$$


and the $b_{p}$ are constants to be determined. Then solutions of

$$
\left(N+\rho \Omega^{2}\right) \chi=0
$$

will yield, through (9), solutions of the Cauchy-Voigt equations.

Upon equating coefficients in (14), we obtain

$$
\begin{aligned}
\gamma_{11}+a_{31}^{2} & =\gamma_{55}+a_{11}^{2}=b_{1}, \\
\gamma_{33}+a_{13}^{2} & =\gamma_{55}+a_{33}^{2}=b_{3}, \\
\gamma_{15}+a_{33} a_{31} & =\gamma_{35}+a_{11} a_{13}=b_{5} .
\end{aligned}
$$

Equations (12) and (16) contain six relations, among the four $a_{i i}$ and the $\gamma_{p q}$, whose consistency requires two relations among the $\gamma_{p q}$. Upon elimination of the $a_{i j}$ from (12) and (16), these relations are found to be

$$
\begin{aligned}
& \left(\gamma_{11}-\gamma_{55}\right)\left(\gamma_{33}-\gamma_{55}\right)=\left(\gamma_{13}+\gamma_{55}\right)^{2}-\left(\gamma_{15}+\gamma_{35}\right)^{2}, \\
& \left(\gamma_{13}+\gamma_{55}\right)\left(\gamma_{15}-\gamma_{35}\right)=\gamma_{35}\left(\gamma_{11}-\gamma_{55}\right)-\gamma_{15}\left(\gamma_{33}-\gamma_{55}\right) .
\end{aligned}
$$
to $(6)$.

If $\gamma_{15}=\gamma_{55}=0$, the second of $A$ is satisfied identically and the first of $A$ reduces

Subject to conditions $A,(12)$ and (16) may be solved for the $a_{i j}$ and $b_{p}$. We note, first, that the first two of (12) and the last two of (16) are satisfied by $a_{13}=a_{31}$ if $b_{5} \neq 0$ and by $a_{11}=-a_{33}$ if $b_{5}=0$. Hence there are two sets of solutions:

$$
\begin{aligned}
& a_{11}= \pm \gamma_{15}\left[\left(\gamma_{11}-\gamma_{33}\right) /\left(\gamma_{15}^{2}-\gamma_{35}^{2}\right)\right]^{1 / 2} \\
& a_{33}= \pm \gamma_{35}\left[\left(\gamma_{11}-\gamma_{33}\right) /\left(\gamma_{15}^{2}-\gamma_{35}^{2}\right)\right]^{1 / 2} \\
& a_{13}=a_{31}= \pm\left[\left(\gamma_{15}^{2}-\gamma_{35}^{2}\right) /\left(\gamma_{11}-\gamma_{33}\right)\right]^{1 / 2} \\
& b_{1}=\gamma_{11}+\left(\gamma_{15}^{2}-\gamma_{35}^{2}\right) /\left(\gamma_{11}-\gamma_{33}\right) \\
& b_{3}=\gamma_{33}+\left(\gamma_{15}^{2}-\gamma_{35}^{2}\right) /\left(\gamma_{11}-\gamma_{33}\right) \\
& b_{5}=\gamma_{15}+\gamma_{35}
\end{aligned}
$$

and

$$
\begin{aligned}
a_{11} & =-a_{33}= \pm i\left[\left(\gamma_{15}^{2}-\gamma_{35}^{2}\right) /\left(\gamma_{11}-\gamma_{33}\right)\right]^{1 / 2} \\
a_{13} & = \pm i \gamma_{35}\left[\left(\gamma_{11}-\gamma_{33}\right) /\left(\gamma_{15}^{2}-\gamma_{35}^{2}\right)\right]^{1 / 2} \\
a_{31} & =\mp i \gamma_{15}\left[\left(\gamma_{11}-\gamma_{33}\right) /\left(\gamma_{15}^{2}-\gamma_{35}^{2}\right)\right]^{1 / 2} \\
b_{1} & =b_{3}=\gamma_{55}-\left(\gamma_{15}^{2}-\gamma_{35}^{2}\right) /\left(\gamma_{11}-\gamma_{33}\right) \\
b_{5} & =0
\end{aligned}
$$

in which either the upper or lower signs may be taken, without loss of generality.

3. Consequences of conditions A. Conditions $A$ take a simpler form when expressed in terms of the elastic compliances, $s_{p a}$, defined by

$$
\epsilon_{p}=s_{p q} \tau_{q} ; \quad s_{p q}=s_{q p} ; \quad p, q=1,3,5 .
$$


From (19) and (3),

$$
\gamma_{p q}=S_{p q} / \Delta,
$$

where $S_{p q}$ is the cofactor of element $s_{p q}$ in the determinant $\Delta=\left|s_{p q}\right|$. When (20) are substituted into conditions $A$, the latter become

$$
s_{11}+s_{33}=2 s_{13}+s_{55}, \quad s_{15}=s_{35}
$$

and we note that conditions $A^{\prime}$ make

$$
\Delta=s_{55}\left(s_{11} s_{33}-s_{13}^{2}-s_{15}^{2}\right) \text {. }
$$

Then, with $\varphi= \pm \chi / \Delta^{1 / 2}$ for (17) and $\psi= \pm i \chi / \Delta^{1 / 2}$ for (18), we find that (9) and (15) become

$$
\begin{aligned}
& u=\left(s_{13}-s_{33}\right) \frac{\partial \varphi}{\partial x}+s_{15} \frac{\partial \varphi}{\partial z}+s_{15} \frac{\partial \psi}{\partial x}+\left(s_{13}-s_{11}\right) \frac{\partial \psi}{\partial z} \\
& w=s_{15} \frac{\partial \varphi}{\partial x}+\left(s_{13}-s_{11}\right) \frac{\partial \varphi}{\partial z}-\left(s_{13}-s_{33}\right) \frac{\partial \psi}{\partial x}-s_{15} \frac{\partial \psi}{\partial z}
\end{aligned}
$$

and

$$
\begin{aligned}
s_{33} \frac{\partial^{2} \varphi}{\partial x^{2}}-2 s_{15} \frac{\partial^{2} \varphi}{\partial x \partial z}+s_{11} \frac{\partial^{2} \varphi}{\partial z^{2}} & =\frac{\rho \Delta}{s_{55}} \frac{\partial^{2} \varphi}{\partial t^{2}} \\
\frac{\partial^{2} \psi}{\partial x^{2}}+\frac{\partial^{2} \psi}{\partial z^{2}} & =\rho s_{55} \frac{\partial^{2} \psi}{\partial t^{2}} .
\end{aligned}
$$

We now rotate to new axes $x_{A}, z_{A}$ according to

$$
x_{A}=x \cos \Psi_{A}-z \sin \Psi_{A}, \quad z_{A}=x \sin \Psi_{A}+z \cos \Psi_{A}
$$

in order to eliminate the cross term in the first of (22). The strain-stress relations, referred to axes $x_{A}, z_{A}$ are written as

$$
\epsilon_{p}^{A}=s_{p q}^{A} \tau_{p}^{A} ; \quad p, q=1,3,5,
$$

where, with $s=\sin \Psi_{A}, c=\cos \Psi_{A}$,

$$
\begin{aligned}
& s_{11}^{A}=s_{11} c^{4}+s_{33} s^{4}+\left(2 s_{13}+s_{55}\right) s^{2} c^{2}-2 s_{15} s c^{3}-2 s_{35} s^{3} c \\
& s_{33}^{A}=s_{11} s^{4}+s_{33} c^{4}+\left(2 s_{13}+s_{55}\right) s^{2} c^{2}+2 s_{15} s^{3} c+2 s_{35} s c^{3} \\
& s_{55}^{A}=4\left(s_{11}+s_{33}-2 s_{13}\right) s^{2} c^{2}+s_{55}\left(c^{2}-s^{2}\right)^{2}+4\left(s_{15}-s_{35}\right)\left(c^{2}-s^{2}\right) s c \\
& s_{13}^{A}=\left(s_{11}+s_{33}-s_{55}\right) s^{2} c^{2}+s_{13}\left(s^{4}+c^{4}\right)+\left(s_{15}-s_{35}\right)\left(c^{2}-s^{2}\right) s c \\
& s_{35}^{A}=2 s_{11} s^{3} c-2 s_{33} s c^{3}+\left(2 s_{13}+s_{55}\right)\left(c^{2}-s^{2}\right) s c+s_{15}\left(3 s^{2} c^{2}-s^{4}\right)+s_{35}\left(c^{4}-3 s^{2} c^{2}\right) \\
& s_{15}^{A}=2 s_{11} s c^{3}-2 s_{33} s^{3} c-\left(2 s_{13}+s_{55}\right)\left(c^{2}-s^{2}\right) s c+s_{15}\left(c^{4}-3 s^{2} c^{2}\right)+s_{35}\left(3 s^{2} c^{2}-s^{4}\right)
\end{aligned}
$$

Now, conditions $A^{\prime}$ make

$$
s_{13}^{A}=s_{13}, \quad s_{55}^{A}=s_{55}, \quad s_{11}^{A}+s_{33}^{A}=s_{11}+s_{33}
$$

and if, in addition, we set

$$
\tan 2 \Psi_{A}=2 s_{15} /\left(s_{33}-s_{11}\right)
$$


we find also

$$
\begin{aligned}
& s_{11}^{A}-s_{33}^{A}=\left(s_{11}-s_{33}\right) \sec 2 \Psi_{A}, \\
& s_{15}^{A}=s_{35}^{A}=0 .
\end{aligned}
$$

With (21) and (24)-(26), the components of displacement, referred to axes $x_{\boldsymbol{A}}$ and $z_{\boldsymbol{A}}$ become

$$
\begin{aligned}
& u_{A}=\left(s_{13}^{A}-s_{33}^{A}\right) \frac{\partial \varphi}{\partial x_{A}}+\left(s_{13}^{A}-s_{11}^{A}\right) \frac{\partial \psi}{\partial z_{A}} \\
& w_{A}=\left(s_{13}^{A}-s_{11}^{A}\right) \frac{\partial \varphi}{\partial z_{A}}-\left(s_{13}^{A}-s_{33}^{A}\right) \frac{\partial \psi}{\partial x_{A}} .
\end{aligned}
$$

Also, the differential equations (22) transform to

$$
\begin{gathered}
s_{33}^{A} \frac{\partial^{2} \varphi}{\partial x_{A}^{2}}+s_{11}^{A} \frac{\partial^{2} \varphi}{\partial z_{A}^{2}}=\rho\left(s_{11}^{A} s_{33}^{A}-s_{13}^{A} s_{13}^{A}\right) \frac{\partial^{2} \varphi}{\partial t^{2}} \\
\frac{\partial^{2} \psi}{\partial x_{A}^{2}}+\frac{\partial^{2} \psi}{\partial z_{A}^{2}}=\rho s_{55}^{A} \frac{\partial^{2} \psi}{\partial t^{2}} .
\end{gathered}
$$

If we were to convert from constants $s_{p q}^{A}$ to $\gamma_{p q}^{A}$ by means of the inverse of (20), we should find that (28) are the same as (8) and that (27) are the same as (7), except for a constant factor. Thus, conditions $A$ reduce the general anisotropic plate to an orthotropic plate satisfying Carrier's condition (6).

4. Slowness vector. Returning to Eqs. (1), we insert the displacements

$$
u=A \exp [i \omega(\xi x+\zeta z-t)], \quad w=C \exp [i \omega(\xi x+\zeta z-t)]
$$

corresponding to straight-crested waves. The resulting characteristic equation, which must be satisfied by the components $\xi, \zeta$ of the slowness vector [4], is

$$
\begin{aligned}
\left(\gamma_{11} \xi^{2}+2 \gamma_{15} \xi \zeta+\gamma_{55} \zeta^{2}-\rho\right)\left(\gamma_{55} \xi^{2}+2 \gamma_{35} \xi \zeta+\gamma_{33} \zeta^{2}-\rho\right) & \\
& -\left[\gamma_{15} \xi^{2}+\left(\gamma_{13}+\gamma_{55}\right) \xi \zeta+\gamma_{35} \zeta^{2}\right]^{2}=0 .
\end{aligned}
$$

With $\xi, \zeta$ as real Cartesian coordinates, (30) is, in general, the equation of a pair of fourlobed curves. However, if conditions $A$ are satisfied, the discriminant of (30) is a perfect square and the curves are a circle, corresponding to the potential $\psi$, and an ellipse, corresponding to $\varphi$, with principal axes oriented in accordance with (25).

The discriminant of $(30)$ is also a perfect square if

$$
\begin{aligned}
& \left(\gamma_{11}-\gamma_{55}\right)\left(\gamma_{13}+\gamma_{55}\right)=2 \gamma_{15}\left(\gamma_{15}-\gamma_{35}\right), \\
& \left(\gamma_{33}-\gamma_{55}\right)\left(\gamma_{13}+\gamma_{55}\right)=2 \gamma_{35}\left(\gamma_{35}-\gamma_{15}\right) .
\end{aligned}
$$

In this case the locus of the tip of the slowness vector is a pair of orthogonal ellipses associated with waves whose properties are described in the following section.

5. Consequences of conditions $\mathbf{B}$. In the case of conditions $B$, we first perform a rotation to new axes

$$
x_{B}=x \cos \Psi_{B}-z \sin \Psi_{B}, \quad z_{B}=x \sin \Psi_{B}+z \cos \Psi_{B} .
$$


Referred to these axes, the stress-strain relation is

$$
\tau_{p}^{B}=\gamma_{p q}^{B} \epsilon_{q}^{B},
$$

where, with $s=\sin \Psi_{B}, c=\cos \Psi_{B}$,

$$
\begin{aligned}
& \gamma_{11}^{B}=\gamma_{11} c^{4}+\gamma_{33} s^{4}+2\left(\gamma_{13}+2 \gamma_{55}\right) s^{2} c^{2}-4 \gamma_{15} s c^{3}-4 \gamma_{35} s^{3} c, \\
& \gamma_{33}^{B}=\gamma_{11} s^{4}+\gamma_{33} c^{4}+2\left(\gamma_{13}+2 \gamma_{55}\right) s^{2} c^{2}+4 \gamma_{15} s^{3} c+4 \gamma_{35} s c^{3}, \\
& \gamma_{55}^{B}=\left(\gamma_{11}+\gamma_{33}-2 \gamma_{13}\right) s^{2} \dot{c}^{2}+\gamma_{55}\left(c^{2}-s^{2}\right)^{2}+2\left(\gamma_{15}-\gamma_{35}\right)\left(c^{2}-s^{2}\right) s c, \\
& \gamma_{13}^{B}=\left(\gamma_{11}+\gamma_{33}-4 \gamma_{55}\right) s^{2} c^{2}+\gamma_{13}\left(s^{4}+c^{4}\right)+2\left(\gamma_{15}-\gamma_{35}\right)\left(c^{2}-s^{2}\right) s c, \\
& \gamma_{35}^{B}=\gamma_{11} s^{3} c-\gamma_{33} s c^{3}+\left(\gamma_{13}+2 \gamma_{55}\right)\left(c^{2}-s^{2}\right) s c+\gamma_{15}\left(3 s^{2} c^{2}-s^{4}\right)+\gamma_{35}\left(c^{4}-3 s^{2} c^{2}\right), \\
& \gamma_{15}^{B}=\gamma_{11} s c^{3}-\gamma_{33} s^{3} c-\left(\gamma_{13}+2 \gamma_{55}\right)\left(c^{2}-s^{2}\right) s c+\gamma_{15}\left(c^{4}-3 s^{2} c^{2}\right)+\gamma_{35}\left(3 s^{2} c^{2}-s^{4}\right) .
\end{aligned}
$$

Now, if we set $\tan 2 \Psi_{B}=\left(\gamma_{13}+\gamma_{55}\right) /\left(\gamma_{35}-\gamma_{15}\right)$ and apply conditions $B$, we find

$$
\gamma_{15}^{B}=\gamma_{35}^{B}=\gamma_{13}^{B}+\gamma_{55}^{B}=0 .
$$

Hence, if $u_{B}$ and $w_{B}$ are the components of displacement referred to axes $x_{B}$ and $z_{B}$, the equations of motion become

$$
\gamma_{11}^{B} \frac{\partial^{2} u_{B}}{\partial x_{B}^{2}}+\gamma_{55}^{B} \frac{\partial^{2} u_{B}}{\partial z_{B}^{2}}=\rho \frac{\partial^{2} u_{B}}{\partial t^{2}}, \quad \gamma_{55}^{B} \frac{\partial^{2} w_{B}}{\partial x_{B}^{2}}+\gamma_{33}^{B} \frac{\partial^{2} w_{B}}{\partial z_{B}^{2}}=\rho \frac{\partial^{2} w_{B}}{\partial t^{2}}
$$

and, consequently, the introduction of displacement-potentials $\varphi$ and $\psi$ is unnecessary.

Equations (31) have the curious property that, in the waves defined by them, the directions of the respective displacements are fixed: independent of the wave-normals. The mechanism which produces this phenomenon may be identified by noting that, under conditions $B$,

$$
\begin{aligned}
& \epsilon_{1}^{B}=\gamma_{33}^{B}\left[\tau_{1}^{B}+\left(\gamma_{55}^{B} / \gamma_{33}^{B}\right) \tau_{3}^{B}\right] /\left(\gamma_{11}^{B} \gamma_{33}^{B}-\gamma_{55}^{B} \gamma_{55}^{B}\right), \\
& \epsilon_{3}^{B}=\gamma_{11}^{B}\left[\tau_{3}^{B}+\left(\gamma_{55}^{B} / \gamma_{11}^{B}\right) \tau_{1}^{B}\right] /\left(\gamma_{11}^{B} \gamma_{33}^{B}-\gamma_{55}^{B} \gamma_{55}^{B}\right) .
\end{aligned}
$$

Hence, since the conditions of positive-definiteness of the strain-energy-density require

$$
\gamma_{11}^{B}>0, \quad \gamma_{33}^{B}>0, \quad \gamma_{55}^{B}>0, \quad \gamma_{11}^{B} \gamma_{33}^{B}-\gamma_{55}^{B} \gamma_{55}^{B}>0,
$$

both Poisson's ratios, $\nu_{1}^{B}=-\gamma_{55}^{B} / \gamma_{33}^{B}$ and $\nu_{3}^{B}=-\gamma_{55}^{B} / \gamma_{11}^{B}$, must be negative and in the ratios indicated. These are properties unlikely to be found in natural crystals.

6. Application of conditions A to quartz. The constants, say $s_{p q}^{\prime \prime}$, of a plate cut from a crystal, at an arbitrary orientation with respect to axes of reference fixed in the crystal, may be expressed in terms of two angles of rotation, say $\Theta$ and $\Phi$, and the constants, say $s_{m n}^{0}, m, n=1, \cdots 6$, associated with the axes of reference. When the expressions for the $s_{p a}^{\prime \prime}$, in terms of the $s_{m n}^{0}, \Theta$ and $\Phi$, are inserted in conditions $A^{\prime}$, in place of the constants $s_{p q}$ appearing there, a pair of simultaneous, transcendental equations in $\Theta$ and $\Phi$ is obtained. If there are any real roots of the pair of equations, they give the orientations of plates whose constants $s_{p q}^{\prime \prime}$ satisfy conditions $A^{\prime}$. 
In the case of quartz, the compliances associated with reference axes $x_{0}, y_{0}, z_{0}$, in accordance with the I.R.E. Standards [5], are

$$
\begin{aligned}
s_{11}^{0}= & s_{22}^{0}=12.77, & & s_{12}^{0}=-1.79, \\
& s_{33}^{0}=9.60, & & s_{13}^{0}=s_{23}^{0}=-1.22, \\
s_{44}^{0}= & s_{55}^{0}=20.04, & & s_{14}^{0}=-s_{24}^{0}=\frac{1}{2} s_{56}^{0}=4.50, \\
s_{66}^{0} & =2\left(s_{11}^{0}-s_{12}^{0}\right), & & \\
s_{15}^{0}= & s_{16}^{0} & = & s_{25}^{0}=s_{26}^{0}=s_{34}^{0}=s_{35}^{0}=s_{36}^{0}=s_{45}^{0}=s_{46}^{0}=0,
\end{aligned}
$$

where the numerical values, in units of $10^{-12}$ (meter) $)^{2} /$ newton, are those given by Bechmann [6].

A rotation of coordinates about the axis of $x_{0}$ to axes

$$
x^{\prime}=x_{0}, \quad y^{\prime}=y_{0} \cos \Theta+z_{0} \sin \Theta, \quad z^{\prime}=-y_{0} \sin \Theta+z_{0} \cos \Theta
$$

gives formulas for the thirteen non-zero compliances $s_{m n}^{\prime}, m ; n=1, \cdots 6$, in terms of the $s_{m n}^{0}$ and $\Theta$, as tabulated by Cady [7].

A further rotation, about the axis of $z^{\prime}$, to axes

$$
x^{\prime \prime}=x^{\prime} \cos \Phi+y^{\prime} \sin \Phi, \quad y^{\prime \prime}=-x^{\prime} \sin \Phi+y^{\prime} \cos \Phi, \quad z^{\prime \prime}=z^{\prime}
$$

gives for the six, of the twenty one, constants which we require:

$$
\begin{aligned}
& s_{11}^{\prime \prime}=s_{11}^{\prime} \cos ^{2} \Phi+s_{22}^{\prime} \sin ^{2} \Phi-\left(s_{11}^{\prime}+s_{22}^{\prime}-2 s_{12}^{\prime}-s_{66}^{\prime}\right) \sin ^{2} \Phi \cos ^{2} \Phi, \\
& s_{33}^{\prime \prime}=s_{33}^{\prime} \\
& s_{55}^{\prime \prime}=s_{44}^{\prime} \sin ^{2} \Phi+s_{55}^{\prime} \cos ^{2} \Phi, \\
& s_{13}^{\prime \prime}=s_{13}^{\prime}+\left(s_{23}^{\prime}-s_{13}^{\prime}\right) \sin ^{2} \Phi, \\
& s_{35}^{\prime \prime}=s_{34}^{\prime} \sin \Phi, \\
& s_{15}^{\prime \prime}=s_{24}^{\prime} \sin \Phi-\left(s_{24}^{\prime}-s_{14}^{\prime}-s_{56}^{\prime}\right) \sin \Phi \cos ^{2} \Phi,
\end{aligned}
$$

in which the $s_{m n}^{\prime}$ are given in terms of the $s_{m n}^{0}$ and $\Theta$ by Cady's [7] formulas.

We now suppose that all of the analysis in Secs. 2 and 3 was performed in the plane of $x^{\prime \prime}$ and $z^{\prime \prime}$, so that the constants $s_{p q}$, appearing there, are replaced by $s_{p q}^{\prime \prime}$. Then, when (32) are inserted in conditions $A^{\prime}$, we find

$$
\begin{aligned}
{\left[s_{14}^{0}\left(3 \sin ^{2} \Theta-4 \cos ^{4} \Theta\right)+2\right.} & \left.k \sin ^{3} \Theta \cos \Theta\right] \sin ^{3} \Phi \\
& -\left[s_{14}^{0}\left(4 \sin ^{4} \Theta-3 \cos ^{2} \Theta\right)+2 k \sin \Theta \cos ^{3} \Theta\right] \sin \Phi=0, \\
{\left[2 s_{14}^{0}\left(3+\cos ^{2} \Theta\right) \sin \Theta \cos \Theta\right.} & \left.-k \sin ^{4} \Theta\right] \sin ^{4} \Phi \\
& +6\left[s_{14}^{0}\left(2 \cos ^{2} \Theta-1\right) \sin \Theta \cos \Theta+k \sin ^{2} \Theta \cos ^{2} \Theta\right] \sin ^{2} \Phi \\
& -2 s_{14}^{0}\left(3+\sin ^{2} \Theta\right) \sin \Theta \cos \Theta-k \cos ^{4} \Theta=0,
\end{aligned}
$$

where $k=s_{11}^{0}+s_{33}^{0}-2 s_{13}^{0}-s_{44}^{0}$. These equations have the four pairs of roots $\Theta$ and $\Phi$ given in the first two rows of the accompanying table. The next six rows give the values of the constants $s_{p a}^{\prime \prime}$ computed from (32). The next row gives the values of $\psi_{A}$ computed from (25), with $s_{p a}$ replaced by $s_{p q}^{\prime \prime}$; and the remaining rows give the values of the constants $s_{p a}^{A}$, referred to the axes $x_{A}$ and $z_{A}$ defined in (23). 
The first column in the table describes the well known " $Z$-cut" of quartz for which the Cauchy-Voigt equations reduce to isotropic form; i.e., the constants satisfy the conditions

$$
s_{11}^{\prime \prime}=s_{33}^{\prime \prime}, \quad s_{55}^{\prime \prime}=2\left(s_{11}^{\prime \prime}-s_{13}^{\prime \prime}\right), \quad s_{15}^{\prime \prime}=s_{35}^{\prime \prime}=0
$$

and Eqs. (4) and (5) apply. This cut has been studied in detail by Petrzilka [8], Bechmann [9] and Ekstein [10]. Modes of vibration of the remaining three cuts may be studied by means of Eqs. (27) and (28).

As anticipated, no cut of quartz has constants of elasticity that satisfy conditions $B$.

\section{TABLE}

Orientations $(\Theta, \Phi, \Psi)$ and compliances $\left(s_{p q}, 10^{-12}\right.$ meter $^{2} /$ newton $)$ of the four cuts of quartz which satisfy conditions $A$.

\begin{tabular}{lcccc}
\hline \hline & I & II & III & IV \\
$\Theta$ & $90^{\circ}$ & $-9.65^{\circ}$ & $18.79^{\circ}$ & $18.79^{\circ}$ \\
$\Phi$ & 0 & 0 & $58.62^{\circ}$ & $-58.62^{\circ}$ \\
$s_{11}^{\prime \prime}$ & 12.77 & 12.77 & 12.52 & 12.52 \\
$s_{33}^{\prime \prime}$ & 12.77 & 9.52 & 9.77 & 9.77 \\
$s_{55}^{\prime \prime}$ & 29.12 & 23.27 & 23.27 & 23.27 \\
$s_{13}^{\prime \prime}$ & -1.79 & -0.49 & -0.49 & -0.49 \\
$s_{35}^{\prime \prime}$ & 0 & 0 & -0.87 & 0.87 \\
$s_{15}^{\prime \prime}$ & 0 & 0 & -0.87 & 0.87 \\
$\Psi_{A}^{\prime \prime}$ & 0 & 0 & $16.17^{\circ}$ & $73.83^{\circ}$ \\
$s_{11}^{A}$ & 12.77 & 12.77 & 12.77 & 9.52 \\
$s_{33}^{A}$ & 12.77 & 9.52 & 9.52 & 12.77 \\
$s_{55}^{A}$ & 29.12 & 23.27 & 23.27 & 23.27 \\
$s_{13}^{A}$ & -1.79 & -0.49 & -0.49 & -0.49 \\
$s_{35}^{A}$ & 0 & 0 & 0 & 0 \\
$s_{15}^{A}$ & 0 & 0 & 0 & 0 \\
\hline
\end{tabular}

\section{REFERENCES}

1. A. L. Cauchy, Exercices de Mathématiques, Paris, 4, 13 (1829); W. Voigt, Lehrbuch der Kristallphysik, 2nd ed., Leipzig, 1928, p. 698

2. G. Lamé, Leçons sur la théorie mathématique de l'élasticité des corps solides, Gauthier-Villars, Paris, 1852, p. 144

3. G. F. Carrier, Quart. Appl. Math. 4, 160 (1946)

4. J. L. Synge, J. Math. and Phys. 35, 323 (1957)

5. Proc. Inst. Radio Eng. 37, 1378 (1949)

6. R. Bechmann, Phys. Rev. 110, 1060 (1958)

7. W. G. Cady, Piezoelectricity, McGraw-Hill Book Co., New York, 1946, p. 77

8. V. Petrzilka, Z. Physik 97, 436 (1935)

9. R. Bechmann, Z. Physik 117, 180 (1941)

10. H. Ekstein, Phys. Rev. 66, 108 (1944) 